\title{
Electron Transport Nanostructures of Conjugated Polymer Films Visualized by Conductive Atomic Force Microscopy
}

\section{$\operatorname{AUTHOR}(S)$ :}

Kondo, Yuya; Osaka, Miki; Benten, Hiroaki; Ohkita, Hideo; Ito, Shinzaburo

\section{CITATION:}

Kondo, Yuya ... [et al]. Electron Transport Nanostructures of Conjugated Polymer Films Visualized by Conductive Atomic Force Microscopy. ACS Macro Letters 2015, 4(9): 879-885

\section{ISSUE DATE:}

2015-08-07

URL:

http://hdl.handle.net/2433/217959

\section{RIGHT:}

This document is the Accepted Manuscript version of a Published Work that appeared in final form in 'ACS Macro Letters', copyright @ $\odot$ American Chemical Society after peer review and technical editing by the publisher. To access the final edited and published work see http://doi.org/10.1021/acsmacrolett.5b00352.; This is not the published version. Please cite only the published version.; この論文は出版社版でありません。引用の際には出版社版をご確認ご利用くだ 


\section{Electron Transport Nanostructures of Conjugated}

\section{Polymer Films Visualized by Conductive Atomic}

\section{Force Microscopy}

Yuya Kondo, ${ }^{1}$ Miki Osaka, ${ }^{1}$ Hiroaki Benten,${ }^{* 1}$ Hideo Ohkita, ${ }^{1}$ Shinzaburo Ito ${ }^{1}$

${ }^{1}$ Department of Polymer Chemistry, Graduate School of Engineering, Kyoto University, Katsura,

Nishikyo, Kyoto 615-8510, Japan

kondo@photo.polym.kyoto-u.ac.jp, osaka@photo.polym.kyoto-u.ac.jp,

benten@photo.polym.kyoto-u.ac.jp, ohkita@photo.polym.kyoto-u.ac.jp,

sito@photo.polym.kyoto-u.ac.jp 


\begin{abstract}
We have successfully measured electron transport nanostructures of conjugated polymer thinfilms by conductive atomic force microscopy, using an air-stable electron-injecting electrode coated with ethoxylated polyethyleneimine. Electron- and hole-transport networks in donor/acceptor polymer blends can be selectively observed by using an appropriately coated electrode. This approach enables us to visualize phase-separated nanostructures of donor/acceptor polymer blends for thin-film electronic devices based on their semiconducting properties.
\end{abstract}

\title{
KEYWORDS
}

Electron transport, Current imaging, Polymer blends, Polymer solar cell, Semiconducting polymer, Polymer acceptor. 


\section{TEXT}

Conjugated polymers have generated increasing academic and industrial interest as key materials for applications in thin-film optoelectronic devices, such as organic solar cells, ${ }^{1,2}$ organic lightemitting diodes, ${ }^{3}$ and organic thin-film transistors. ${ }^{2,4}$ Their excellent semiconducting properties and compatibility with printing- or solution-based large-scale processing methods have opened new possibilities for low-cost and flexible plastic electronics. ${ }^{5}$ For these polymer-based devices, the nanoscopic and mesoscopic morphology of the active layers strongly influences the physical processes such as charge generation and transport. ${ }^{6-9}$ Therefore, high-resolution techniques for characterizing the electrical nanostructures of active layers are of prime importance for further material and device improvement.

Conductive atomic force microscopy (C-AFM) is a useful method for directly observing the charge-transport properties in both the lateral and vertical directions of conjugated polymer films with a high resolution on the order of nanometers. This technique has been employed to study the relationship between the nanostructures and the electrical functions of various $p$-type donor polymer films. ${ }^{10-19}$ For $n$-type acceptor polymers, on the other hand, there are few C-AFM studies because of a limited variation of acceptor polymers and the lack of air-stable electroninjecting electrodes. However, the development of acceptor polymers is strongly desired not only for thin-film transistors, ${ }^{20}$ but also for polymer solar cells composed of donor and acceptor polymers. $^{21}$

In 2009, Facchetti and his co-workers reported a novel acceptor polymer, P(NDI2OD-T2) (Polyera ActivInk ${ }^{\mathrm{TM}}$ N2200), which exhibits high electron mobility and air-stability. ${ }^{22}$ This polymer is now widely used as a promising material for electron transport; consequently, the semiconducting nanostructures of N2200 neat and blend films have received a great deal of 
attention, ${ }^{23-29}$ directed at the design of next-generation high-performance $n$-type acceptor polymers. As for the electron-injecting electrodes, Kippelen and his co-workers recently reported a method to produce air-stable low work function (WF) electrodes by using polymer surface modifiers such as $80 \%$ ethoxylated polyethyleneimine (PEIE) and branched polyethyleneimine (PEI). ${ }^{30}$ An interfacial dipole formed by the PEIE and PEI layer is known to reduce the WF of electrodes substantially, which can reduce the electron injection energy barrier into organic semiconductors. ${ }^{30}$

In this study, we developed a method, based on the C-AFM approach, to observe the local electron transport properties of acceptor polymers by using air-stable cathode substrates coated with a PEIE layer. With this approach, we visualized the nanostructures for electron transport of N2200 films. Moreover, we also imaged both electron and hole transport networks formed in blend films with a donor polymer, poly(3-hexylthiophene) (P3HT).

Regioregular P3HT (regioregularity $>90.0 \%, M_{\mathrm{w}}=51,000 \mathrm{~g} \mathrm{~mol}^{-1}$, polydispersity index (PDI) $=2.4)$ was purchased from Aldrich Chemical Co. N2200 was purchased from Polyera Co. $\left(M_{\mathrm{w}}=\right.$ 99,750 $\mathrm{g} \mathrm{mol}^{-1}$, PDI = 3.5). A PEIE aqueous solution $\left(35-40 \mathrm{wt} \%, M_{\mathrm{w}}=70,000 \mathrm{~g} \mathrm{~mol}^{-1}\right)$ was purchased from Aldrich Chemical Co. The aqueous solution was further diluted with 2methoxyethanol to a weight concentration of 0.04 and $2 \mathrm{wt} \%$; they were then used for spincoating. The chemical structures of these polymers are shown in Figure 1. Indium-tin-oxide (ITO) substrates (Geomatec Co., Ltd., FLAT ITO) were sequentially washed by ultrasonication in toluene, acetone, and ethanol for 15 min each, and then dried under a $\mathrm{N}_{2}$ flow. These cleaned substrates were further treated with a $\mathrm{UV}-\mathrm{O}_{3}$ cleaner (Nippon Laser \& Electronics Lab., NLUV253S) for 30 min. The PEIE-coated ITO electrode (PEIE electrode) was prepared as follows: 
a PEIE layer was prepared on the ITO substrate by spin-coating using a 0.04 wt\% 2methoxyethanol solution at a spin rate of $5000 \mathrm{rpm}$ for $60 \mathrm{~s}$ and then dried at $100{ }^{\circ} \mathrm{C}$ for $10 \mathrm{~min}$ in ambient air. ${ }^{30}$ The PEIE electrode for the electron-current imaging of the N2200 neat film was prepared using a $2 \mathrm{wt} \%$ 2-methoxyethanol solution, followed by rinsing with ultrapure water and drying with $\mathrm{N}_{2} .^{30}$ An ITO electrode, coated with poly(3,4-ethylenedioxythiophene):poly(4-

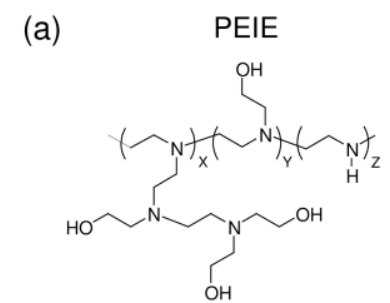

(b)

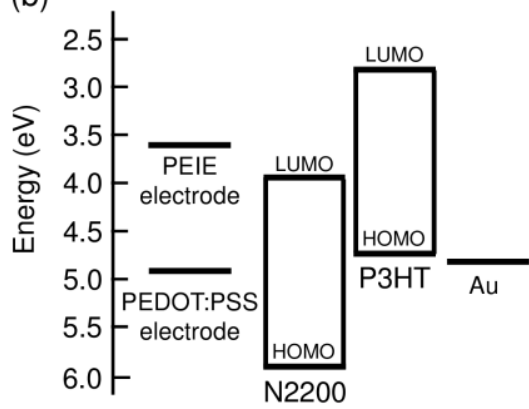

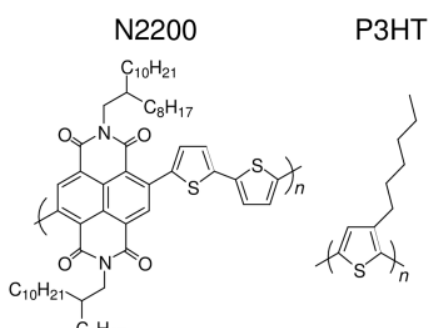

(c)
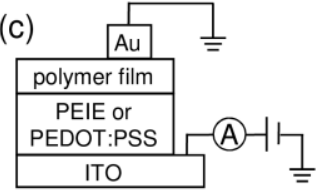

(d)

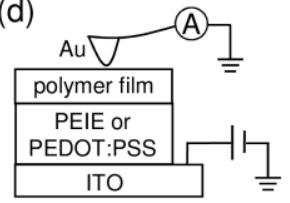

Figure 1. (a) Chemical structures of PEIE, N2200, and P3HT. (b) Energy level diagram of electrodes and polymers. Each energy level is taken from the literature: a work function of 3.6 $\mathrm{eV}$ for ITO/PEIE, ${ }^{30} 4.9 \mathrm{eV}$ for ITO/PEDOT:PSS, ${ }^{30} 4.8 \mathrm{eV}$ for $\mathrm{Au} ;{ }^{31} \mathrm{HOMO}$ energies of 5.9 eV for N2200 and $4.7 \mathrm{eV}$ for P3HT; and LUMO energies of $3.9 \mathrm{eV}$ for N2200 and $2.7 \mathrm{eV}$ for P3HT. ${ }^{32-34}$ The HOMO energies were evaluated by photoelectron yield spectroscopy, and the LUMO energy of P3HT was estimated by adding the optical bandgap energy, calculated from the $0-0$ transition, to the HOMO enery. ${ }^{32,33}$ The LUMO energy of N2200 was determined from cyclic voltammetry measurements performed on the thin film. ${ }^{34}$ (c) Schematic of the macroscopic $J-V$ measurement of polymer films. (d) Schematic of the local current measurement by C-AFM: A negative (positive) bias voltage was applied to the bottom electrode, and the electron (hole) current through the polymer film to the AFM tip was detected. 
styrenesulfonate) (PEDOT:PSS; H.C. Stark, PH-500), hereafter referred to as the PEDOT:PSS electrode, was prepared as follows: a PEDOT:PSS layer was prepared on the ITO substrate by spin-coating and then dried at $140{ }^{\circ} \mathrm{C}$ for $10 \mathrm{~min}$ in ambient air. For neat film preparation, N2200 was dissolved to form a 1 wt\% solution in chlorobenzene, and P3HT was dissolved to form a $1 \mathrm{wt} \%$ solution in chloroform. For blend film preparation, N2200 and P3HT were codissolved at a 50/50 weight ratio to form a $1 \mathrm{wt} \%(0.5 \mathrm{wt} \%$ each) solution in chlorobenzene. The neat films of N2200 and P3HT, and the blend films of P3HT/N2200 were separately prepared by spin-coating from these solutions onto the PEIE and the PEDOT:PSS electrodes in a $\mathrm{N}_{2}$-filled glove box. The energy level diagram of the electrodes and the polymers is shown in Figure $1 \mathrm{~b}$. For the measurements of macroscopic current density-voltage $(J-V)$ characteristics, a Au top electrode (thickness of $50 \mathrm{~nm}$, surface area of $0.07 \mathrm{~cm}^{2}$ ) was deposited by vacuum evaporation on top of the N2200 (film thickness of $80 \mathrm{~nm}$ ) and the P3HT (film thickness of 90-100 nm) neat films. The $J-V$ characteristics were measured by applying bias to the bottom PEIE and PEDOT:PSS electrodes in the dark (Figure 1c), using a direct-current voltage and current source/monitor (Advantest, R6243). The C-AFM measurements were performed using a Shimadzu SPM-9600 with a Au-coated silicon probe (PPP-CONTAu; NANO-SENSORS ${ }^{\mathrm{TM}}$, tip radius $<50 \mathrm{~nm}$, spring constant $=0.2 \mathrm{~N} \mathrm{~m}^{-1}$ ) under a $\mathrm{N}_{2}$ atmosphere using a controlledenvironment chamber (CH-III, Shimadzu, Japan). The local current-voltage (I-V) characteristics were obtained for P3HT (film thickness of 90-100 nm) and N2200 (film thickness of 60-70 nm) neat films by recording current magnitudes as a function of applied voltage between the bottom electrode and the top AFM probe at a specified sample position (Figure 1d). The C-AFM current images were obtained for N2200 neat and P3HT/N2200 blend films (film thickness of $60 \mathrm{~nm}$ ) by recording current magnitudes with a constant sample bias applied to the 
bottom electrode while the AFM probe was scanned over the surface. The topographical information of the sample was recorded simultaneously with the current magnitudes.

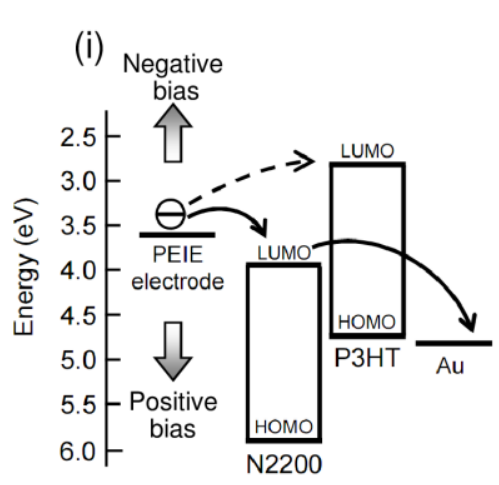

(ii)

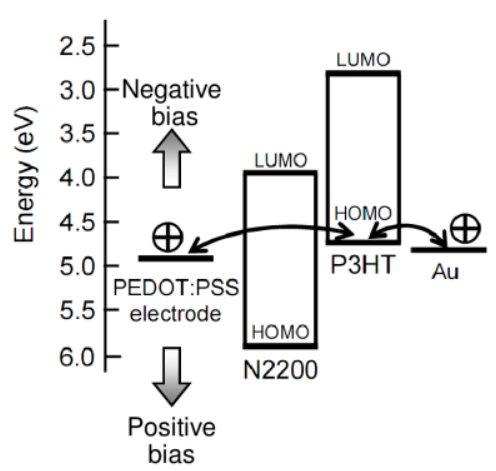

Macroscopic $J-V$ characteristics

(a)

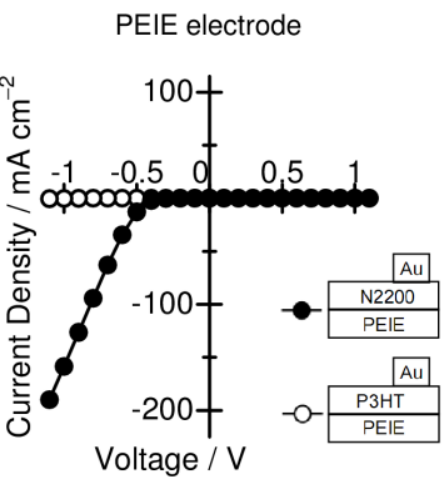

(b)

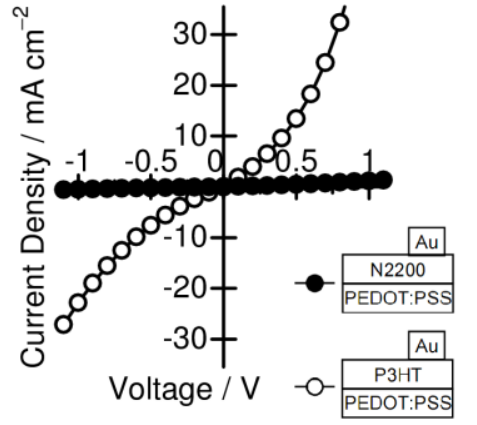

C-AFM local $I-V$ characteristics

(c) PEIE electrode

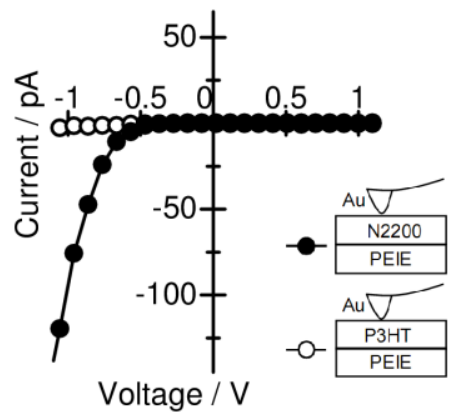

(d)

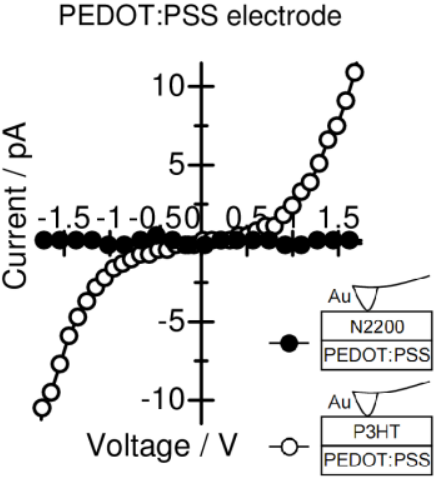

Figure 2. Macroscopic $J-V$ characteristics of N2200 (closed circles) and P3HT (open circles) films prepared on the (a) PEIE electrode and (b) PEDOT:PSS electrode. For both $J-V$ measurements, bias voltages were applied to the PEIE and PEDOT:PSS electrodes. C-AFM local $I-V$ characteristics of $\mathrm{N} 2200$ (closed circles) and P3HT (open circles) films prepared on the (c) PEIE electrode and (d) PEDOT:PSS electrode. For both $I-V$ measurements, bias voltages were applied to the PEIE and PEDOT:PSS electrodes. (i) At negative bias, electrons are injected from the PEIE electrode into the LUMO of N2200 and extracted at the Au electrode, producing electron current. (ii) At positive (negative) bias, holes are injected from PEDOT:PSS $(\mathrm{Au})$ electrodes into the HOMO of P3HT and extracted at Au (PEDOT:PSS) electrode, producing hole current. 
We first measured the macroscopic $J-V$ characteristics of $\mathrm{N} 2200$ and P3HT neat films on the PEIE and PEDOT:PSS electrodes to examine charge injection properties from the PEIE and PEDOT:PSS electrodes into N2200 and P3HT. The closed circles in Figures 2a and $2 \mathrm{~b}$ show the $J-V$ characteristics observed for the N2200 films on each electrode. For N2200 films on the PEIE electrode (closed circles, Figure 2a), the current from the N2200 was observed only with a negative bias voltage applied to the PEIE electrode. This is ascribed to the electron current injected from the PEIE electrode because there exists a large energy barrier $(\sim 1.1 \mathrm{eV})$ for hole injection from the Au anode into the highest occupied molecular orbital (HOMO) of N2200 as shown in Figure 1b. On the other hand, the WF of the PEIE electrode is reported to be $3.6 \mathrm{eV},{ }^{30}$ lying above the energy level of the lowest unoccupied molecular orbital (LUMO) of N2200. Therefore, our result indicates that the WF of ITO is decreased successfully by the PEIE coating, enabling electrons to be injected into the LUMO of N2200 as illustrated in the inset in Figure 2. For N2200 films on the PEDOT:PSS electrode (closed circles, Figure 2b), no current was observed from the N2200. This is because electron injection into N2200 is difficult owing to a large energy barrier of approximately $1.0 \mathrm{eV}$ between the WF of the PEDOT:PSS electrode and the LUMO of N2200 as shown in Figure $1 \mathrm{~b}$.

The open circles in Figures $2 \mathrm{a}$ and $2 \mathrm{~b}$ show the $J-V$ characteristics observed for the P3HT films. For P3HT films on the PEIE electrode (open circles, Figure 2a), no current was observed at either bias polarity, which is very different from the response in the case of N2200. This result implies that almost no electrons are injected into the LUMO of P3HT from the PEIE electrode because of an electron injection barrier of approximately $0.9 \mathrm{eV}$. Moreover, the PEIE can serve as a hole-blocking layer that prevents the holes in the P3HT from being collected at the ITO interface. For P3HT films on the PEDOT:PSS electrode (open circles, Figure 2b), current was 
observed for both bias polarities; this is ascribed to hole current because efficient hole injection occurs into the HOMO of P3HT from both the PEDOT:PSS bottom (at applied positive voltages) and the Au top electrodes (at applied negative voltages) as illustrated in the inset in Figure 2.

Next, we measured the local $I-V$ characteristics of the N2200 and P3HT films on the PEIE and PEDOT:PSS electrodes by using a Au conductive probe. As shown in Figures $2 \mathrm{c}$ and $2 \mathrm{~d}$, the local $I-V$ characteristics were similar to the corresponding macroscopic results shown in Figures $2 \mathrm{a}$ and $2 \mathrm{~b}$. This demonstrates that both the electron transport properties in films of an acceptor polymer and the hole transport properties of a donor polymer can be estimated qualitatively regardless of whether the Au electrode or the Au-coated AFM probe is used.
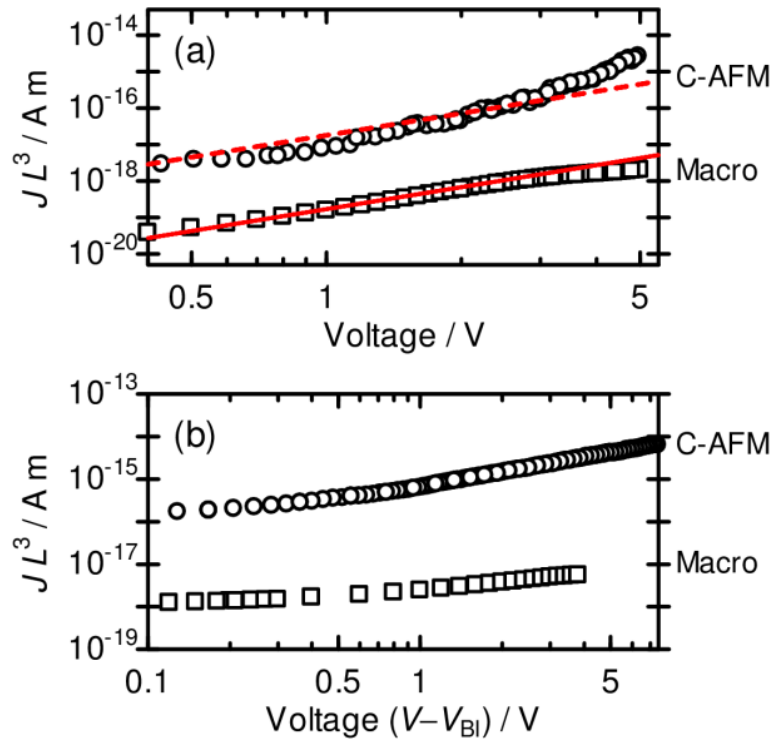

Figure 3. (a) Macroscopic (squares) and C-AFM local (circles) $J-V$ characteristics obtained at negative bias voltages for P3HT neat films on the PEDOT:PSS electrode. The solid and dashed lines are fits to the $J-V$ data using Eq. 1 and Eq. 2, respectively, to extract the hole mobilities. (b) Macroscopic (squares) and C-AFM local (circles) $J-V$ characteristics obtained at negative bias voltages for N2200 neat films on the PEIE electrode. The applied voltage $V$ is corrected for the built-in potential $V_{\mathrm{BI}}{ }^{41}$ The C-AFM local current density was calculated from the $I-V$ data shown in Figures $2 \mathrm{c}$ and $2 \mathrm{~d}$ assuming the tip-sample contact area as $311 \mathrm{~nm}^{2},{ }^{38}$ and the resultant current densities are normalized for each film thickness. 
For the P3HT neat films on the PEDOT:PSS electrode (Figures $2 \mathrm{~b}$ and $2 \mathrm{~d}$ ), the small or nonexistent threshold voltage and the parabolic-like variation of the current with the applied voltage are consistent with previously reported results. ${ }^{15,35}$ This indicates high hole injection, likely by an Ohmic contact between the electrodes and the sample. Therefore, we extracted the hole mobility $\mu_{\mathrm{h}}$ of the P3HT by fitting the $J-V$ characteristics in the space-charge-limited current (SCLC) regime to a Mott-Gurney equation, ${ }^{35,36}$

$$
J_{\mathrm{SCLC}}=\frac{9}{8} \varepsilon_{0} \varepsilon_{\mathrm{r}} \mu \frac{V^{2}}{L^{3}},
$$

where $J$ is the current density, $\mu$ is the charge carrier mobility, $V$ is the applied voltage, $L$ is the thickness of the film, $\varepsilon_{0}$ is the vacuum permittivity, and $\varepsilon_{\mathrm{r}}$ is the dielectric constant of the film (we assumed $\left.\mathcal{E}_{\mathrm{r}}=3\right)^{35}$. The straight solid line in Figure 3(a) is fit of Eq. 1 to the macroscopic $J-$ $V$ data measured at negative bias voltages (squares). The fit to the $J-V$ data gave hole mobility ( $\left.\mu_{\text {Macro }}\right)$ of $5.7 \times 10^{-5} \mathrm{~cm}^{2} \mathrm{~V}^{-1} \mathrm{~s}^{-1}$, which is in good agreement with the values of $\mu_{\mathrm{h}}$ reported in the literatures $\left(\mu_{\mathrm{h}}=1.3 \times 10^{-5}-3.3 \times 10^{-4} \mathrm{~cm}^{2} \mathrm{~V}^{-1} \mathrm{~s}^{-1}\right)^{35,37}$. On the other hand, as shown by the circles in Figure 3(a), the local hole current densities obtained by C-AFM measurements are two orders of magnitude larger than the current densities obtained by macroscopic device measurements. ${ }^{38}$ This means that two orders of larger mobility value than that of $\mu_{\text {Macro }}$ would be extracted when using the eq 1 to fit the C-AFM data. Such an overestimation of $\mu_{\mathrm{h}}$ has been reliably corrected by Ginger et al., who introduced a semiempirical scaling factor defined by the ratio of the tip-sample contact area diameter $d$ to sample thickness $L \cdot{ }^{37}$ They proposed a modified Mott-Gurney equation to fit the SCLC current in C-AFM:

$$
J_{\text {SCLC }}=\alpha \varepsilon_{0} \varepsilon_{\mathrm{r}} \mu \frac{V^{2}}{L^{3}} \delta\left(\frac{L}{d}\right)^{1.6 \pm 0.1}
$$


where $\alpha$ is the prefactor determined from finite element modeling to account for the nonuniform electrical field ( $\alpha=8.2$ in place of $9 / 8$ for the Mott-Gurney law for planar electrodes), and the constant $\delta=7.8 \pm 1$ is an empirical dimensionless parameter that accounts for the difference between mobilities derived from C-AFM measurements and measurements using macroscopic planar electrodes. ${ }^{37}$ When using $d=19.8 \mathrm{~nm}$ and $L=100 \mathrm{~nm}$, (see the Supporting Information), the fit of eq 2 to the C-AFM data, shown by the dashed line in Figure 3a, yielded a hole mobility $\left(\mu_{\mathrm{C}-\mathrm{AFM}}\right)$ of $0.79 \times 10^{-5} \mathrm{~cm}^{2} \mathrm{~V}^{-1} \mathrm{~s}^{-1}$.

Figure 3(b) shows the macroscopic (squares) and C-AFM local (circles) $J-V$ characteristics of the N2200 neat films on the PEIE electrode at negative bias voltages; the $J-V$ curves show similar power-law dependences of current with the applied voltages. Moreover, the local electron current densities measured by C-AFM are larger than macroscopic current densities by more than two orders of magnitude, which is similar to the results of the $J-V$ characteristics for P3HT hole current.

In summary, the use of the PEIE electrode as a cathode enables the characterization of the local electron transport properties of N2200 by the C-AFM measurements. Moreover, the hole current of P3HT can be detected by using the PEDOT:PSS electrode. Therefore, when this approach is applied to the P3HT/N2200 blend films, the electron current through the N2200 phase should be detected selectively with the PEIE electrode, and the hole current through the P3HT phase should be detected selectively with the PEDOT:PSS electrode, as described below.

The C-AFM was applied to perform the local electrical characterization of the P3HT/N2200 blend films. We first employed the PEIE electrode to obtain the electron-current image. Figures $4 \mathrm{a}$ and $4 \mathrm{~b}$ display topographic and electron-current images, respectively, for the blend 

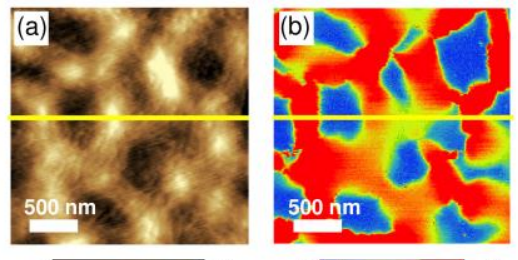

Height / nm

(c)
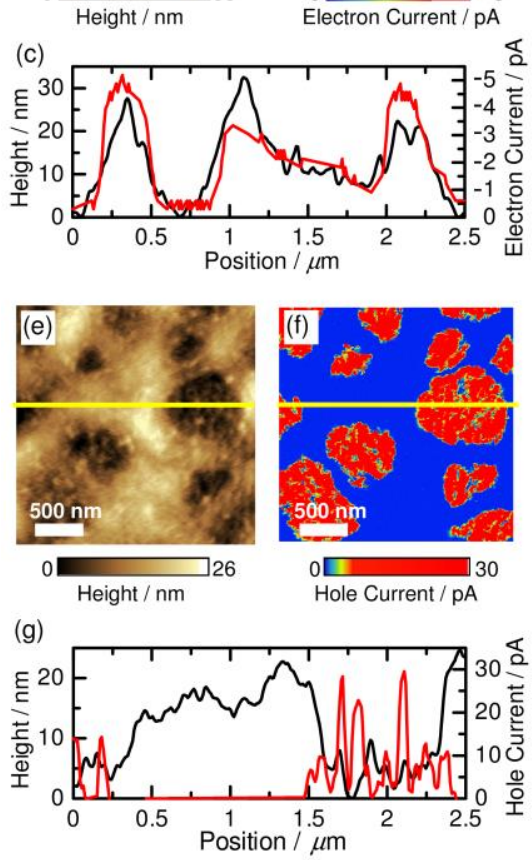

(d)

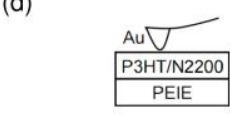

- High-lying region

-O- Low-lying region

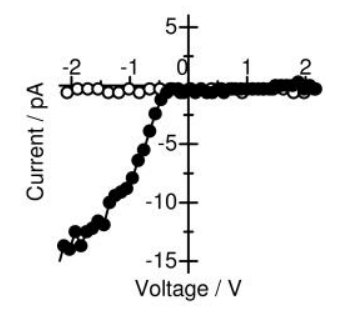

(h)
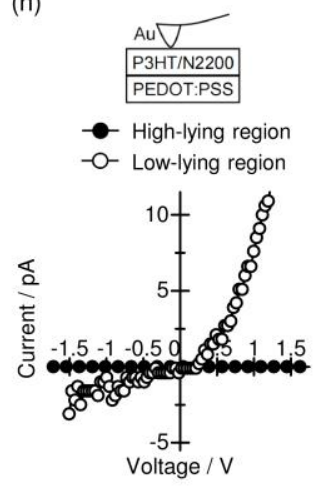

Figure 4. C-AFM (a) topographic and (b) electron-current images $\left(2.5 \times 2.5 \mu \mathrm{m}^{2}\right)$ of the P3HT/N2200 blend film spin-coated on the PEIE electrode. (c) The cross-sectional profiles of height (black line, left) and current flow (red line, right) along the horizontal line ruled in the images of the panel (a) and (b), respectively. These electron-current maps and the corresponding topographic images were measured simultaneously at $-2 \mathrm{~V}$ applied to the PEIE electrode. (d) The representative $I-V$ profiles observed at the high-lying (closed circles) and low-lying (open circles) regions in the blend film. C-AFM (e) topographic and (f) holecurrent images $\left(2.5 \times 2.5 \mu \mathrm{m}^{2}\right)$ of the same blend film spin-coated on the PEDOT:PSS electrode. (g) The cross-sectional profiles of height (black line, left) and current flow (red line, right) along the horizontal line ruled in the images of the panel (e) and (f), respectively. These hole-current maps and the corresponding topographic images were measured simultaneously at $+2 \mathrm{~V}$ applied to the PEDOT:PSS electrode. (h) The representative $I-V$ profiles observed at the high-lying (closed circles) and low-lying (open circles) regions in the blend film. 
film on the PEIE electrode. For both topographic and electron-current images, a very similar blend morphology composed of sea and island structures on a submicrometer scale was observed. Figure $4 \mathrm{c}$ shows the cross-sectional profiles of the height (black line) and electron current (red line) along the line across each image. These cross-sectional profiles show that the regions with high electron-current flow correspond mainly to the high-lying structures. Considering that only the N2200 allows the electron current to flow under this device configuration, we ascribe the high-lying structures to the N2200-rich phase. Figure 4d shows representative $I-V$ characteristics observed at the high-lying structures and low-lying regions. The $I-V$ profile at the high-lying structures (closed circles) exhibits current flow only with a negative applied voltage, which well represents the characteristics of N2200 neat film. On the other hand, the lack of current response in the low-lying regions (open circles) implies that these regions correspond to the P3HT-rich phase. We next employed the PEDOT:PSS electrode to obtain the hole-current image. Figures $4 \mathrm{e}$ and $4 \mathrm{f}$ display topographic and hole-current images, respectively. Figure $4 \mathrm{~g}$ shows the cross-sectional profiles of the height (black line) and hole current (red line) along the line across each image. As shown in the figure, hole current was observed selectively in the lowlying regions. Further, the representative $I-V$ profile at the low-lying regions (open circles, Figure 4h) exhibits current flow with both negative and positive applied voltages, which is qualitatively consistent with the result of the P3HT neat film. We note that the $I-V$ profile of the hole current in P3HT/N2200 blend films is different from that observed for P3HT neat films: the $I-V$ response is asymmetric with lower current at negative bias. The lower hole injection from the AFM tip into the blend film might be attributed to a high contact resistance present between the tip and the sample. On the other hand, the lack of current response at the high-lying structures (closed circles, Figure 4h) supports our assignment that these structures correspond to 
the N2200-rich phase. We therefore conclude that the high-lying and low-lying regions correspond to the N2200-rich and P3HT-rich phases, respectively.

In summary, the C-AFM approach using the PEIE electrode can visualize the electron-transport domains of N2200 in the blend film with P3HT. Moreover, the electron- and hole-current images obtained by the PEIE and PEDOT:PSS electrodes provide complementary information about the phase-separated structures of the P3HT/N2200, donor/acceptor, polymer blends. Further, the local $I-V$ characterization by C-AFM will allow us to study the electron and hole conductivity and mobility of the individual microstructures, ${ }^{16,37,42}$ which could never be estimated by macroscopic $J-V$ measurements.

Previously, Nguyen et al. obtained electron-current images and the nanoscale electron-transport properties for a blend film composed of $\mathrm{P} 3 \mathrm{HT}$ as a donor and a fullerene derivative (PCBM) as an acceptor. In their work, they used a Mg-coated glass substrate and a Mg-coated AFM tip to measure the electron current. ${ }^{16}$ In contrast, our approach uses a device comprising a PEIEcoated ITO electrode and Au-coated AFM tip, which has the same structure as an inverted-type polymer solar cell in which a PEIE-coated electrode is used as the cathode for electron collection and a high-WF metal (such as Au or Ag) is used as the anode to collect holes. Therefore, our method can be performed on donor/acceptor blend samples used in solar cells, which would enable us to directly examine the correlation between the electron transport nanostructures and macroscopic photovoltaic performances of the blends.

Another key feature of the current-contrast imaging lies in its ability to reveal fine details of nanoscale morphology of the blend, which are obscured in topographic imaging. Figures 5a and 
$5 \mathrm{~b}$ show high-magnification topographic and electron-current images of the P3HT/N2200 blend film. The electron-current image enables us to distinguish the compositions sharply, visualizing the percolation paths for electron transport of N2200 in the blend. In addition, electrontransporting structures with a size of tens of nanometers are visualized even in the low-lying region; these are marked by the circles in Figures $5 \mathrm{a}$ and $5 \mathrm{~b}$. The electron current would be assigned to the N2200 that is sequestered as a minor component within the P3HT-rich phase during the process of phase-separation. The hole-current image (Figure 5d) also visualizes the percolation paths for hole transport with a size of tens of nanometers in the high-lying N2200rich phase; it is marked by the circle in Figures $5 \mathrm{c}$ and $5 \mathrm{~d}$. As shown in these images, the
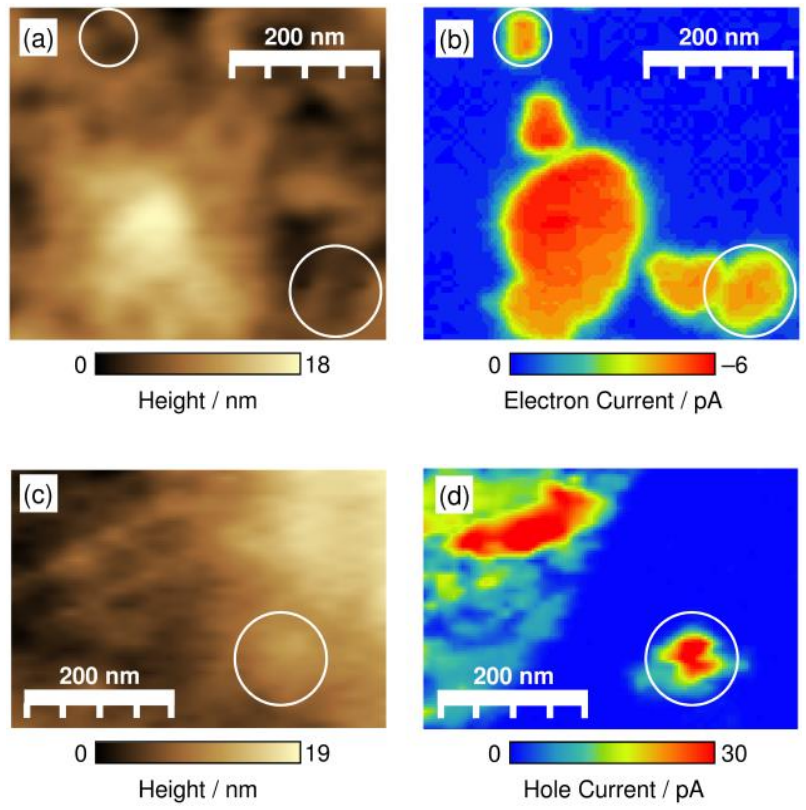

Figure 5. C-AFM (a) topographic and (b) electron-current images of the P3HT/N2200 blend film spin-coated on the PEIE electrode. C-AFM (c) topographic and (d) hole-current images of the P3HT/N2200 blend film spin-coated on the PEDOT:PSS electrode. These current maps and the corresponding topographic images were measured simultaneously at $-1 \mathrm{~V}$ applied to the PEIE electrode and at $+2 \mathrm{~V}$ applied to the PEDOT:PSS electrode. 
nanoscale percolation paths for electron transport (hole transport) developed within the donorrich (acceptor-rich) domains are also characterized by this approach. Such fine details of phaseseparated nanostructures provided by C-AFM current images are of prime importance for the development of optoelectronic devices, especially in polymer solar cells, where a phase separation of 10-20-nm domain size and efficient percolation paths throughout the layer thickness are required. ${ }^{43,44}$

Finally, we show that the high-resolution C-AFM electron-current imaging makes it feasible to visualize the electrical nanostructures for thin films of polymer acceptors. Figures $6 \mathrm{a}$ and $6 \mathrm{~b}$ show the surface topographical image and the corresponding electron-current image, respectively, for an N2200 neat film on the PEIE electrode. The electron-current image clearly depicts ribbon-like features with length ranging from several hundred nanometers to a few micrometers, which differ from the surface topography, showing nanofibrous morphology similar to the results previously observed. ${ }^{24,25}$ The electron-current image is rather similar to the long-range ordering structures on the micrometer length scale that were observed in a study using transmission electron microscopy (TEM). ${ }^{26}$ Further, it is verified from cross-section analysis that there is not a strong correlation between the topographical structures and the current magnitudes associated with each position (Figure 6c). These results suggest that the electron-current image represents the bulk morphology of N2200 film. The inhomogeneous current magnitudes at each position are probably associated with the difference in the density of locally ordered structures of N2200 in the film. 


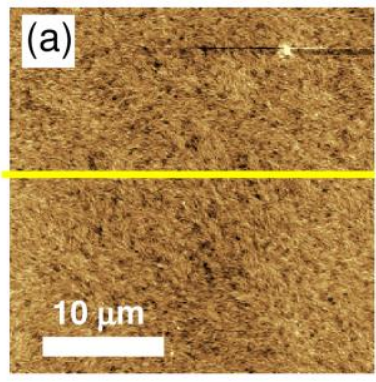

$0 \square 12$

Height / nm

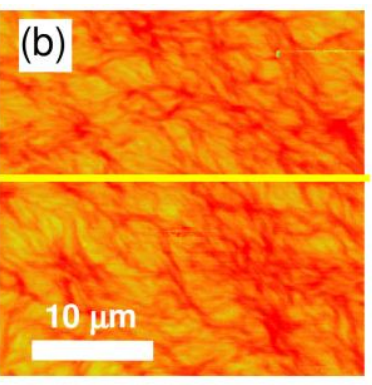

$0 \square-14$

Electron Current / pA

(c)

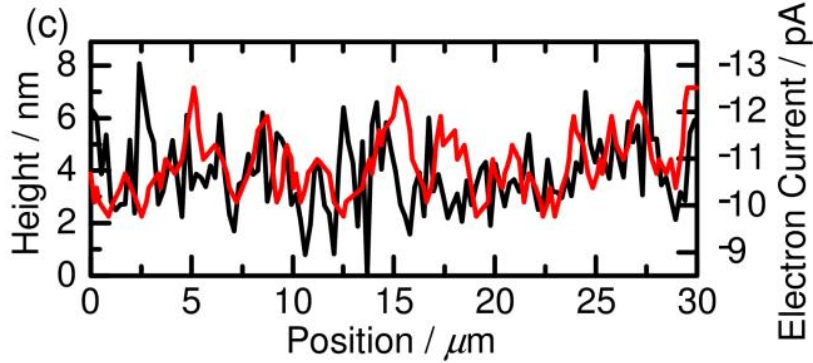

Figure 6. C-AFM (a) topographic and (b) electron-current images $\left(30 \times 30 \mu \mathrm{m}^{2}\right)$ of the N2200 neat film on the PEIE electrode. (c) The cross-sectional profiles of the height (black line, left) and current flow (red line right) along the horizontal line ruled in the images of the panel (a) and (b), respectively. These current maps and the corresponding topographic images were measured simultaneously at $-2 \mathrm{~V}$ applied to the PEIE electrode. The scale bar corresponds to a length of $10 \mu \mathrm{m}$.

In conclusion, we have established a useful approach for imaging the electron transport nanostructures of conjugated polymer films by C-AFM, using an air-stable cathode coated with a PEIE interlayer. This approach has numerous advantages as a high-resolution scanning probe technique for the study of conjugated polymer films as follows.

1. The current images can reveal fine details of electron-transport structures with the size of tens of nanometers in the blends, which are not easily distinguished in the topographical image.

2. Electron- and hole-transport networks in donor/acceptor polymer blends can be distinguished 
in a mutually complementary manner by using an appropriate coating on the electrode.

3. The local $I-V$ measurements will enable us to extract an electron conductivity and mobility of the nanostructures, which could never be estimated by macroscopic $J-V$ measurements.

4. The bulk morphology for electron transport can be brought to light for the thin film of polymer acceptors.

These advantages underline the capability of C-AFM to electrically resolve nanostructures of conjugated polymers, contributing to further understanding of the mechanisms for the excellent electron transport and the creation of photovoltaic functions. 
Corresponding Author

Hiroaki Benten

Tel.: +81 753832614 Fax: +81 753832617 Email: benten@photo.polym.kyoto-u.ac.jp

\section{Author Contributions}

The manuscript was written through contributions of all authors. All authors have given approval to the final version of the manuscript.

\section{ACKNOWLEDGMENT}

This work was partly supported by the CREST program from the Japan Science and Technology Agency (JST) and Technology Agency, and JSPS KAKENHI Grant Number 26288060.

\section{Supporting Information Available}

$* * * * *$

This material is available free of charge via the Internet at http://pubs.acs.org 


\section{REFERENCES}

(1) Cheng, Y. J.; Yang, S. H.; Hsu, C. S. Chem. Rev. 2009, 109, 5868-5923.

(2) Facchetti, A. Chem. Mater. 2011, 23, 733-758.

(3) Grimsdale, A. C.; Chan, K. L.; Martin, R. E.; Jokisz, P. G.; Holmes, A. B. Chem. Rev. 2009, 109, 897-1091.

(4) Sirringhaus, H. Adv. Mater. 2014, 26, 1319-1335.

(5) Søndergaard, R. R.; Hösel, M.; Krebs, F. C. J. Polym. Sci., Part B: Polym. Phys. 2013, 51, $16-34$.

(6) Moos. E. J. Phys.: Condens. Matter 2002, 14, 12235-12260.

(7) Kline, R. J.; Mcgehee, M. D. J. Macromol. Sci., Polym. Rev. 2006, 46, 27-45.

(8) Salleo, A. Mater. Today 2007, 10, 38-45.

(9) Mori, D.; Benten, H.; Ohkita, H.; Ito, S. Adv. Energy Mater. 2015, 5, 1500304.

(10) Alexeev, A.; Loos, J.; Koetse, M. M. Ultramicroscopy 2006, 106, 191-199.

(11) Groves. C.; Reid, O. G.; Ginger, D. S. Acc. Chem. Res. 2010, 43, 612-620.

(12) Mativetsky, J. M.; Loo, Y.-L, Samori, P. J. Mater. Chem. C 2014, 2, 3118-3128.

(13) Moerman, D.; Sebaihi, N.; Kaviyil, S. E.; Leclère, P. Lazzaroni, R.; Douhéret, O. Nanoscale 2014, 6, 10596-10603.

(14) Lin, H.-N.; Lin, H.-L.; Wang, S.-S.; Yu, L.-S.; Perng, G.-Y.; Chen, S.-A.; Chen, S.-H. Appl. Phys. Lett. 2002, 81, 2572-2574.

(15) Douhéret. O.; Swinnen, A.; Breselge, M. Severen, I. V.; Lutsen, L.; Vanderzande, D.; Manca, J. Microelectron. Eng. 2007, 84, 431-436.

(16) Dante, M.; Peet, J.; Nguyen, T. Q. J. Phys. Chem. C 2008, 112, 7241-7249.

(17) Chen, M. C.; Hung, W. C.; Su. A. C.; Chen, S. H.; Chen, S. A. J. Phys. Chem. B 2009, 


\section{$113,11124-11133$.}

(18) Lee, L. T.; Ito, S.; Benten, H.; Ohkita, H.; Mori, D. AMBIO 2012, 41, 135-137.

(19) Osaka, M.; Benten, H.; Lee, L.-T.; Ohkita, H.; Ito, S. Polymer 2013, 54, 3443-3447.

(20) Usta, H.; Facchetti, A.; Marks, T. J. Acc. Chem. Res. 2011, 44, 501-510.

(21) Facchetti, A. Mater. Today 2013, 16, 123-132.

(22) Yan, H.; Chen, Z.; Zheng, Y.; Newman, C.; Guinn, J. R.; Dötz, F.; Kastler, M.; Facchetti, A. Nature 2009, 457, 679-686.

(23) Rivnay, J.; Toney, M. F.; Zheng, Y.; Kauvar, I. V.; Chen, Z.; Wagner, V.; Facchetti, A.; Salleo, A. Adv. Mater. 2010, 22, 4359-4363.

(24) Rivnay, J. Steyrleuthner, R.; Jimison, L. H.; Casadei, A.; Chen, Z.; Toney, M. F.; Facchetti, A.; Neher, D.; Salleo, A. Macromolecules 2011, 44, 5246-5255.

(25) Schuettfort, T.; Huettner, S.; Lilliu, S.; Macdonald, J. E.; Thomsen, L.; McNeill, C. R. Macromolecules 2011, 44, 1530-1539.

(26) Takacs, C. J.; Treat, N. D.; Krämer, S.; Chen, Z.; Facchetti, A.; Chabinyc, M. L.; Heeger, A. J. Nano Lett. 2013, 13, 2522-2527.

(27) Steyrleuthner, R.; Pietro, R. D.; Collins, B. A.; Polzer, F.; Himmelberger, S.; Schubert, M.; Chen, Z.; Zhang, S.; Salleo, A.; Ade, H.; Facchetti, A.; Neher, D. J. Am. Chem. Soc. 2014, 136, 4245-4256.

(28) Yan, H.; Collins, B. A.; Gann, E.; Wang, C.; Ade, H.; McNeill, C. R. ACS Nano 2012, 6, $677-688$.

(29) Schubert, M.; Dolfen, D.; Frisch, J.; Roland, S.; Steyrleuthner, R.; Stiller, B.; Chen, Z.; Scherf, U. Koch, N.; Facchetti, A.; Neher, D. Adv. Energy Mater. 2012, 2, 369-380.

(30) Zhou, Y.; Fuentes-Hernandez, C.; Shim, J.; Meyer, J.; Giordano, A. J.; Li, H.; Winget, P.; 
Papadopoulos, T.; Cheun, H.; Kim, J.; Fenoll, M.; Dindar, A.; Haske, W.; Najafabadi, E.;

Khan, T. M.; Sojoudi, H.; Barlow, S.; Graham, S.; Bredas, J.-L.; Marder, S. R.; Kahn, A.

Kippelen, B. Science 2012, 336, 327-332.

(31) Asadi, K.; Boer, T. G.; Blom, P. W. M.; Leeuw, D. M. Adv. Funct. Mater. 2009, 19, $3173-3178$.

(32) Mori, D.; Benten. H.; Okada, I.; Ohkita, H.; Ito, S. Adv. Energy Mater. 2014, 4, 1301006.

(33) Mori, D. Benten. H.; Kosaka, J.; Ohkita, H.; Ito, S.; Miyake, K. ACS Appl. Mater.

Interfaces 2011, 3, 2924-2927.

(34) Chen, Z.; Zheng, Y.; Yan, H.; Facchetti, A. J. Am. Chem. Soc. 2009, 131, 8-9.

(35) Goh, C.; Kline, J. R.; McGehee, M. D.; Kadnikova, E. N.; Fréchet, J. M. J. Appl. Phys. Lett. 2005, 86, 122110.

(36) Shen, Y.; Hosseini, A. R.; Wong, M. H.; Malliaras, G. G. ChemPhysChem. 2004, 5, 1625.

(37) Reid, O. G.; Munechika, K.; Ginger, D. S. Nano Lett. 2008, 8, 1602-1609.

(38) Estimation of hole mobility from the C-AFM local $I-V$ characteristics requires conversion from current to current density. The contact area between the tip and the sample was estimated to be $311 \mathrm{~nm}^{2}$ according to the method in Ref. 37 by Reid et al. (see the Supporting Information).

(39) Wetzelaer, G. J. A. H.; Kuik, M.; Olivier, Y.; Lemaur, V.; Cornil, J.; Fabiano, S.; Loi, M. A.; Blom, P. W. M. Phys. Rev. B 2012, 86, 165203.

(40) Malliaras, G. G.; Salem, J. R.; Brock, P. J.; Scott, C. Phys. Rev. B 1998, 58, R13411R13414.

(41) The value of $V_{\mathrm{BI}}$ was evaluated to be $1.2 \mathrm{eV}$ from the difference of the work functions 
between ITO/PEIE (3.6 eV) and Au (4.8 eV).

(42) MacDonald, G. A.; Veneman, P. A.; Placencia, D.; Armstrong, N. R. ACS Nano 2012, 6, 9623-9636.

(43) Heeger, A. J. Adv. Mater. 2014, 26, 10-28.

(44) McNeill, C. R. Energy Environ. Sci. 2012, 5, 5653-5667. 
Insert Table of Contents Graphic and Synopsis Here

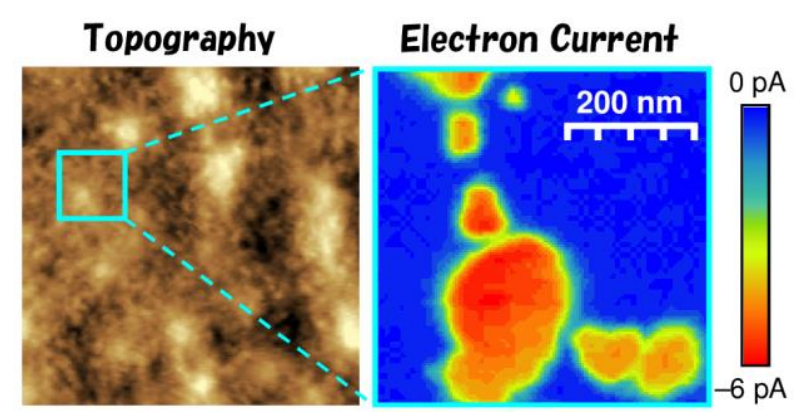

